\title{
LIFTING GOTTLIEB SETS AND DUALITY
}

\author{
YEON SOO YOON
}

(Communicated by Frederick R. Cohen)

\begin{abstract}
Let $p: E_{f} \rightarrow X$ be a fibration induced by a map $f: X \rightarrow Y$ from the path space fibration $\varepsilon: P Y \rightarrow Y$. Let $g: A \rightarrow X$ be cyclic. When does $g$ lift to a map $A \rightarrow E_{f}$ which is cyclic? We give an answer of this question for arbitrary $A$ and $Y$. Also, we give an answer in the dual situation.
\end{abstract}

\section{INTRODUCTION}

A based map $f: A \rightarrow X$ is cyclic [8] if there exists a map $\phi: X \times A \rightarrow X$ such that $\phi j$ is homotopic to $\nabla(1 \vee f)$, where $j: X \vee A \rightarrow X \times A$ is the inclusion and $\nabla: X \vee X \rightarrow X$ is the folding map. The Gottlieb set denoted $G(A, X)$ is the set of all homotopy classes of cyclic maps from $A$ to $X$. Dually, a based map $f: X \rightarrow A$ is cocyclic [8] if there exists a map $\theta: X \rightarrow X \vee A$ such that $j \theta$ is homotopic to $(1 \times f) \Delta$, where $j: X \vee A \rightarrow X \times A$ is the inclusion and $\Delta: X \rightarrow X \times X$ is the diagonal map. The dual Gottlieb set $D G(X, A)$ is the set of all homotopy classes of cocyclic maps from $X$ to $A$. In this paper we consider the following problem and its dual. Let $f: X \rightarrow Y$ be a map and $P Y$ the space of paths in $Y$ which begin at ${ }^{*}$. Let $\varepsilon: P Y \rightarrow Y$ be the fibration given by $\varepsilon(\eta)=\eta(1)$. Let $p: E_{f} \rightarrow X$ be the fibration induced by $f$ from $\varepsilon$, that is, $E_{f}=\{(x, \eta) \in X \times P Y \mid f(x)=\varepsilon(\eta)\}$ is the pullback of $f: X \rightarrow Y$ and $\varepsilon: P Y \rightarrow Y$. Let $g: A \rightarrow X$ be cyclic, that is, there is a map $\phi: X \times A \rightarrow X$ such that $\phi j \sim \nabla(1 \vee g)$, where $j: X \vee A \rightarrow X \times A$ is the inclusion. When does $g$ lift to a map $A \rightarrow E_{f}$ which is cyclic? This can be achieved, if there is a map $\hat{\phi}: E_{f} \times A \rightarrow E_{f}$ such that $\hat{\phi}_{\mid E_{f}} \sim 1_{E_{f}}$ and $\phi(p \times 1)=p \hat{\phi}$. In case $A=S^{n}$ and $Y$ is an Eilenberg-Mac Lane space, Gottlieb [1, Theorem 6.3], has given a necessary and sufficient condition for the existence of this $\hat{\phi}$. In case $A$ is arbitrary and $Y$ is a product of Eilenberg-Mac Lane spaces, Halbhavi and Varadarajan [2] have given a necessary and sufficient condition for the existence of such a $\hat{\phi}$. In case $A$ is arbitrary and $Y$ is an $H$-space, Hoo [4] has also given a necessary and sufficient condition for the existence of such a $\hat{\phi}$. We can obtain a necessary and sufficient condition for the existence of such a $\hat{\phi}$ with arbitrary $A$ and $Y$, and we show that Hoo's necessary and sufficient condition

Received by the editors May 29, 1991.

1991 Mathematics Subject Classification. Primary 55R05, 55P05.

Key words and phrases. Cyclic maps, cocyclic maps, principal fibrations, principal cofibrations.

The author was partially supported by KOSEF 891-0103-008-2 and TGRC during this research. 
follows from ours. Our method is a modification of Haslam's which was used to study Postnikov systems and $G$-spaces. We now consider the dual situation. Let $f: X \rightarrow Y$ be a map and $c X$ the reduced cone of $X$. There is a cofibration $X \stackrel{l}{\rightarrow} c X \rightarrow \Sigma X$, where $l(x)=[x, 1]$. Let $i: Y \rightarrow C_{f}$ be the cofibration induced by $f$ from $l$, that is, $C_{f}=c X \amalg Y / l(x) \sim f(x)$ is the pushout of $f: X \rightarrow Y$ and $l: X \rightarrow c X$. Let $g: Y \rightarrow A$ be cocyclic, that is, there is a map $\theta: Y \rightarrow Y \vee A$ such that $j \theta \sim(1 \times g) \Delta$, where $j: Y \vee A \rightarrow Y \times A$ is the inclusion. When does $g$ extend to a map $C_{f} \rightarrow A$ which is cocyclic? When there is a map $\hat{\theta}: C_{f} \rightarrow C_{f} \vee A$ such that $p_{1} j^{\prime} \hat{\theta} \sim 1_{C_{f}}$ and $(i \vee 1) \theta=\hat{\theta} i$, this can be achieved, where $j^{\prime}: C_{f} \vee A \rightarrow C_{f} \times A$ is the inclusion and $p_{1}: C_{f} \times A \rightarrow C_{f}$ is the projection. In case $A$ is arbitrary and $X$ is a wedge product of Moore spaces, Halbhavi and Varadarajan [2] have given a necessary and sufficient condition for the existence of this $\hat{\theta}$. In case $A$ is arbitrary and $X$ is a co- $H$-space, Hoo [4] has given a necessary and sufficient condition for the existence of such a $\hat{\theta}$. We can obtain a necessary and sufficient condition for the existence of such a $\hat{\theta}$ with arbitrary $A$ and $X$, and we show that Hoo's necessary and sufficient condition follows from ours. All our spaces will be homotopy type of connected locally finite $\mathrm{CW}$ complexes. We assume also that spaces have nondegenerate base points. All homotopies and maps are to respect base points. The base point as well as the constant map will be denoted by *. Also, we denote by $[X, Y]$ the set of homotopy classes of pointed maps $X \rightarrow Y$. The identity map of space will be denoted by 1 when it is clear from the context. $\Sigma X$ and $\Omega X$ denote the reduced suspension and the loop space of $X$ respectively. The adjoint functor from the group $[\Sigma X, Y]$ to the group $[X, \Omega Y]$ will be denoted by $\tau$.

\section{Lifting Gottlieb SETS}

Let $f: X \rightarrow Y$ be a map and $P Y$ the space of paths in $Y$ which begin at *. Let $\varepsilon: P Y \rightarrow Y$ be the fibration given by evaluating a path at its end point. Let $p: E_{f} \rightarrow X$ be the fibration induced by $f$ from $\varepsilon$. That is, $E_{f}$ is the pullback of $f: X \rightarrow Y$ and $\varepsilon: P Y \rightarrow Y$;

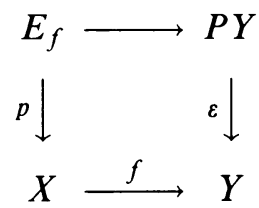

where $E_{f}=\{(x, \eta) \in X \times P Y \mid f(x)=\varepsilon(\eta)\}, p(x, \eta)=x$. In fact, the fibration $p: E_{f} \rightarrow X$ is principal.

The following two lemmas are standard.

Lemma 2.1. A map $g: A \rightarrow X$ can be lifted to a map $A \rightarrow E_{f}$ if and only if $f g \sim *$.

Lemma 2.2 [3]. Given maps $g_{k}: A_{k} \rightarrow E_{f}, k=1,2$, and $g: A_{1} \times A_{2} \rightarrow E_{f}$ satisfying $p g_{\mid A_{k}} \sim p g_{k}, k=1,2$, then there is a map $h: A_{1} \times A_{2} \rightarrow E_{f}$ such that $p h=p g$ and $h_{\mid A_{k}} \sim g_{k}, k=1,2$.

Theorem 2.3. Let $g: A \rightarrow X$ be cyclic, that is, there is a map $\phi: X \times A \rightarrow X$ such that $\phi j \sim \nabla(1 \vee g)$, where $j: X \vee A \rightarrow X \times A$ is the inclusion. Then there 
exists a map $\hat{\phi}: E_{f} \times A \rightarrow E_{f}$ such that $\hat{\phi}_{\mid E_{f}} \sim 1_{E_{f}}$ and the diagram

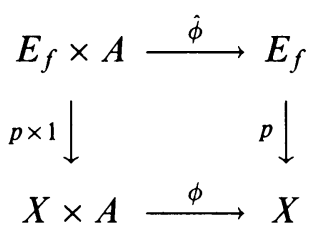

commutes if and only if $f \phi(p \times 1) \sim *$.

Proof. If such a $\hat{\phi}$ exists, we have, from Lemma 2.1, that $f \phi(p \times 1) \sim *$. Conversely, suppose $f \phi(p \times 1) \sim *$. By Lemma 2.1, there is a map $\phi^{\prime}: E_{f} \times A \rightarrow$ $E_{f}$ such that $p \phi^{\prime}=\phi(p \times 1)$. Then $p \phi_{\mid E_{f}}^{\prime}=\phi(p \times 1)_{\mid E_{f}} \sim p 1_{E_{f}}$. Thus we have, from Lemma 2.2 , that there is a map $\hat{\phi}: E_{f} \times A \rightarrow E_{f}$ such that $p \hat{\phi}=p \phi^{\prime}=\phi(p \times 1), \hat{\phi}_{\mid E_{f}} \sim 1_{E_{f}}$, and $\hat{\phi}_{\mid A} \sim \phi_{\mid A}^{\prime}$. This proves the theorem.

Consider the following diagram where each square homotopy commutes and each column is the Puppe sequences of the cofibration:

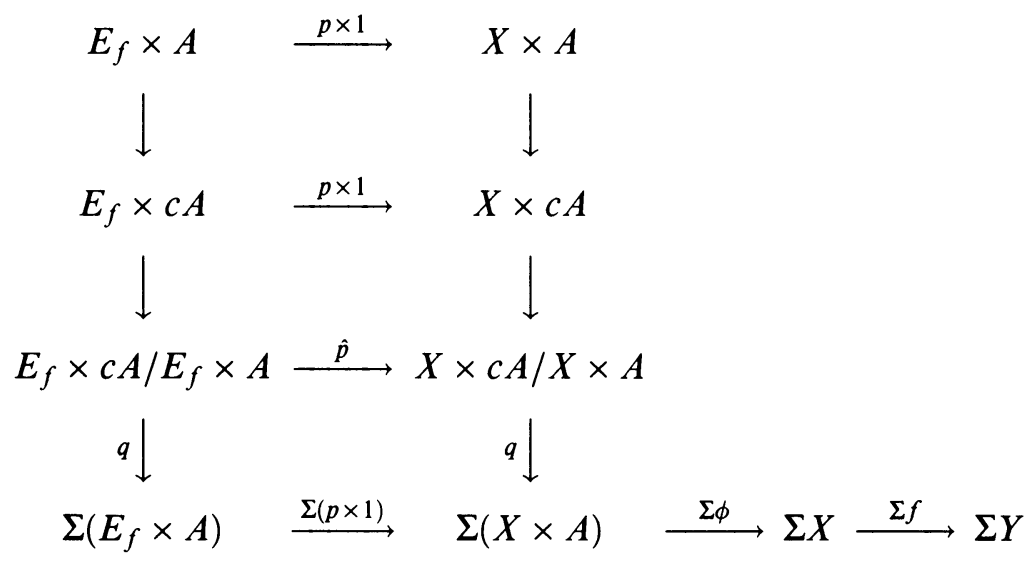

where $\hat{p}$ is induced by $p \times 1$.

Corollary 2.4 [4, Theorem 1]. Let $Y$ be an $H$-space and $g: A \rightarrow X$ cyclic, that is, there is a map $\phi: X \times A \rightarrow X$ such that $\phi j \sim \nabla(1 \vee g)$, where $j: X \vee A \rightarrow X \times A$ is the inclusion. Then there exists a map $\hat{\phi}: E_{f} \times A \rightarrow E_{f}$ such that $\hat{\phi}_{\mid E_{f}} \sim 1_{E_{f}}$ and $p \hat{\phi}=\phi(p \times 1)$ if and only if $\Sigma(f \phi) q \hat{p} \sim *$.

Proof. From Theorem 2.3, it is sufficient to show that $\Sigma(f \phi) q \hat{p} \sim *$ if and only if $f \phi(p \times 1) \sim *$. If $f \phi(p \times 1) \sim *$, then $\Sigma(f \phi) q \hat{p} \sim \Sigma(f \phi(p \times 1)) q \sim$ $*$. Suppose $\Sigma(f \phi) q \hat{p} \sim *$. Since $E_{f} \times A \stackrel{(1 \times l)}{\longrightarrow} E_{f} \times c A \rightarrow E_{f} \times c A / E_{f} \times$ $A$ is a cofibration, there is an exact sequence $\rightarrow\left[\Sigma\left(E_{f} \times c A\right), \Sigma Y\right] \stackrel{(\Sigma(1 \times l))^{*}}{\longrightarrow}$ $\left[\Sigma\left(E_{f} \times A\right), \Sigma Y\right] \stackrel{q^{*}}{\rightarrow}\left[E_{f} \times A / E_{f} \times A, \Sigma Y\right] \rightarrow$. Since $q^{*}(\Sigma(f \phi(p \times 1))) \sim$ $\Sigma(f \phi) q \hat{p} \sim *$ and $\operatorname{Ker} q^{*}=\operatorname{Im}(\Sigma(1 \times l))^{*}$, there is a map $k: \Sigma\left(E_{f} \times c A\right) \rightarrow \Sigma Y$ such that $k_{\mid \Sigma\left(E_{f} \times A\right)}=\Sigma(f \phi(p \times 1))$. Taking adjoints, we get a map $E_{f} \times c A \rightarrow$ $\Omega \Sigma Y$ extending $e^{\prime} f \phi(p \times 1)$, where $e^{\prime}=\tau\left(1_{\Sigma Y}\right)$. Since $Y$ is an $H$-space, there is a map $r: \Omega \Sigma Y \rightarrow Y$ such that $r e^{\prime} \sim 1_{Y}$. Hence we have a map $H: E_{f} \times A \times I \rightarrow Y$ satisfying $H(, 0)=f \phi(p \times 1)$ and $H(,, 1)=f p p_{1}$, where $p_{1}: E_{f} \times A \rightarrow E_{f}$ is the projection. From Lemma $2.1, H(,, 1)=f p p_{1} \sim *$. Thus $f \phi(p \times 1) \sim *$. 
We can also obtain the following proposition. The proof of Proposition 2.5 is similar to that of Theorem 2.3. So we will omit the proof.

Proposition 2.5. Let $g: A \rightarrow E_{f}$ be a map such that $p g: A \rightarrow X$ is cyclic, that is, there is a map $\phi: X \times A \rightarrow X$ such that $\phi j \sim \nabla(1 \vee p g)$, where $j: X \vee A \rightarrow X \times A$ is the inclusion. Then there exists a map $\hat{\phi}: E_{f} \times A \rightarrow E_{f}$ such that $\hat{\phi}_{\mid E_{f}} \sim 1_{E_{f}}, \hat{\phi}_{\mid A} \sim g$, and $p \hat{\phi}=\phi(p \times 1)$ if and only if $f \phi(p \times 1) \sim *$.

Corollary 2.6. Let $[A, X]=0$. Then, for any map $f: X \rightarrow Y,\left[A, E_{f}\right]=$ $G\left(A, E_{f}\right)$.

Proof. Consider the map $\phi: X \times A \rightarrow X$ is given by $\phi(x, a)=x$. Then $\phi(p \times 1)=p p_{1}$, where $p_{1}: E_{f} \times A \rightarrow E_{f}$ is the projection. That is, $\phi(p \times 1)$ : $E_{f} \times A \rightarrow X$ can be lifted to the map $p_{1}: E_{f} \times A \rightarrow E_{f}$. Thus we know, from Lemma 2.1, that $f \phi(p \times 1) \sim *$. Let $[g] \in\left[A, E_{f}\right]$. Since $p_{*}([g]) \in[A, X]=$ $0, p g \sim *$. Thus $\phi j \sim \nabla(1 \vee *) \sim \nabla(1 \vee p g)$. From the fact $f \phi(p \times 1) \sim *$ and Proposition 2.5, $[g] \in G\left(A, E_{f}\right)$.

Remark 2.7. Consider the map $\psi: E_{f} \times \Omega Y \rightarrow E_{f}$ given by $\psi((x, \eta), \omega)=$ $(x, \eta+\omega)$, where + denotes the usual product of two paths. Then the inclusion $i: \Omega Y \rightarrow E_{f}$ is cyclic. Thus the induced map $i_{*}$ maps $[A, \Omega Y]$ into $G\left(A, E_{f}\right)$ [8]. Therefore, Corollary 2.6 can also be obtained from the long exact sequence of homotopy sets for the fibration $\Omega Y \rightarrow E_{f} \rightarrow X$.

\section{Extending DUAL GotTlieb SETS}

We now consider the dual situation. There is a well-known cofibration $X \stackrel{!}{\rightarrow}$ $c X \rightarrow \Sigma X$, where $l(x)=[x, 1], c X$ is the reduced cone, and $\Sigma X$ is the reduced suspension. Given a map $f: X \rightarrow Y$, consider the cofibration $Y \stackrel{i}{\rightarrow}$ $C_{f} \rightarrow \Sigma X$ induced by $f: X \rightarrow Y$ from $l$. That is, $C_{f}$ is the pushout of $f: X \rightarrow Y$ and $\imath: X \rightarrow c X:$

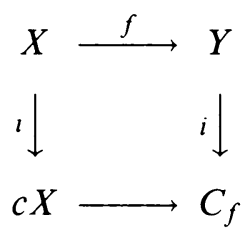

where $C_{f}$ is the mapping cone of $f$, the space obtained from $c X \amalg Y$ by identifying $[x, 1] \in c X$ with $f(x), i(y)=y$. In fact, the cofibration $Y \stackrel{i}{\rightarrow}$ $C_{f} \rightarrow \Sigma X$ is a principal cofibration [7]. Thus there is a map $\phi: C_{f} \rightarrow \Sigma X \vee C_{f}$ such that the following diagram is commutative:

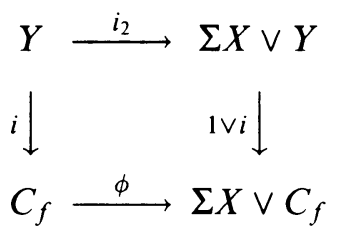

where $i_{2}$ is the inclusion and $\phi: C_{f} \rightarrow \Sigma X \vee C_{f}$ is given by $\phi(y)=(*, y)$, $\phi([x, t])=(\langle x, 2 t\rangle, *), 0 \leq t \leq 1 / 2, \phi([x, t])=(*,[x, 2 t-1]), 1 / 2 \leq t \leq 1$.

The following two lemmas are standard. 
Lemma 3.1 [6, Proposition 2.34, p. 25]. A map $g: Y \rightarrow A$ can be extended to $C_{f}$ (there is a map $h: C_{f} \rightarrow A$ such that $h i=g$ ) if and only if $g f \sim *$.

Lemma 3.2 [7]. Let $g_{1}, g_{2}: C_{f} \rightarrow A$ be maps. Then $g_{1} i \sim g_{2} i$ if and only if there is a map $\gamma: \Sigma X \rightarrow A$ such that $g_{1} \sim \nabla\left(\gamma \vee g_{2}\right) \phi$, where $\phi: C_{f} \rightarrow \Sigma X \vee C_{f}$ is given by $\phi(y)=(*, y), \phi([x, t])=(\langle x, 2 t\rangle, *), 0 \leq t \leq 1 / 2, \phi([x, t])=$ $(*,[x, 2 t-1]), 1 / 2 \leq t \leq 1$.

Lemma 3.3. Let $g_{k}: C_{f} \rightarrow A_{k}$ be maps, $k=1,2$, and $g: C_{f} \rightarrow A_{1} \vee A_{2} a$ map such that $p_{k} j g i \sim g_{k} i, k=1,2$, where $j: A_{1} \vee A_{2} \rightarrow A_{1} \times A_{2}$ is the inclusion and $p_{k}: A_{1} \times A_{2} \rightarrow A_{k}, k=1,2$, are projections. Then there is a map $h: C_{f} \rightarrow A_{1} \vee A_{2}$ such that $g i=h i$ and $p_{k} j h \sim g_{k}, k=1,2$.

Proof. By Lemma 3.2, there are maps $\gamma_{k}: \Sigma X \rightarrow A_{k}$ such that $g_{k} \sim$ $\nabla\left(\gamma_{k} \vee p_{k} j g\right) \phi, k=1,2$. Let $\gamma=\left(\gamma_{1} \vee \gamma_{2}\right) \mu: \Sigma X \rightarrow A_{1} \vee A_{2}$, where $\mu: \Sigma X \rightarrow$ $\Sigma X \vee \Sigma X$ is the co- $H$-structure. Consider the map $h=\nabla(\gamma \vee g) \phi: C_{f} \rightarrow A_{1} \vee A_{2}$. Then $h i=g i$ follows from the fact $\phi i=(1 \vee i) i_{2}$. Moreover,

$$
\begin{aligned}
p_{k} j h & =p_{k} j \nabla(\gamma \vee g) \phi=\nabla\left(p_{k} \vee p_{k}\right)(j \vee j)(\gamma \vee g) \phi \\
& =\nabla\left(p_{k} j \gamma \vee p_{k} j g\right) \phi \sim \nabla\left(\gamma_{k} \vee p_{k} j g\right) \phi \sim g_{k} .
\end{aligned}
$$

This proves the lemma.

Now we have the following theorem which is the dual of Theorem 2.3.

Theorem 3.4. Let $g: Y \rightarrow A$ be cocyclic, that is, there is a map $\theta: Y \rightarrow Y \vee A$ such that $j \theta \sim(1 \times g) \Delta$, where $j: Y \vee A \rightarrow Y \times A$ is the inclusion and $\Delta: Y \rightarrow Y \times Y$ is the diagonal map. Then there exists a map $\hat{\theta}: C_{f} \rightarrow C_{f} \vee A$ such that $p_{1} j^{\prime} \hat{\theta} \sim 1_{C_{f}}$ and the diagram

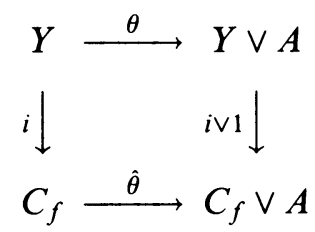

commutes if and only if $(i \vee 1) \theta f \sim *$, where $j^{\prime}: C_{f} \vee A \rightarrow C_{f} \times A$ is the inclusion and $p_{1}: C_{f} \times A \rightarrow C_{f}$ is the projection.

Proof. If such a $\hat{\theta}$ exists, we have, from Lemma 3.1, that $(i \vee 1) \theta f \sim *$. Conversely, suppose $(i \vee 1) \theta f \sim *$. By Lemma 3.1, there is a map $\theta^{\prime}: C_{f} \rightarrow$ $C_{f} \vee A$ such that $\theta^{\prime} i=(i \vee 1) \theta$. Then $p_{1} j^{\prime} \theta^{\prime} i=p_{1} j^{\prime}(i \vee 1) \theta=p_{1}(i \times 1) j \theta \sim$ $p_{1}(i \times g) \Delta=1_{C_{f}} i$. Thus we have, from Lemma 3.3, that there is a map $\hat{\theta}$ : $C_{f} \rightarrow C_{f} \vee A$ such that $\hat{\theta} i=\theta^{\prime} i=(i \vee 1) \theta, p_{1} j^{\prime} \hat{\theta} \sim 1_{C_{f}}$, and $p_{2} j^{\prime} \hat{\theta} \sim p_{2} j^{\prime} \theta^{\prime}$. This proves the theorem.

Consider the following diagram where each square homotopy commutes and 
each column is the Puppe sequence of the fibration:

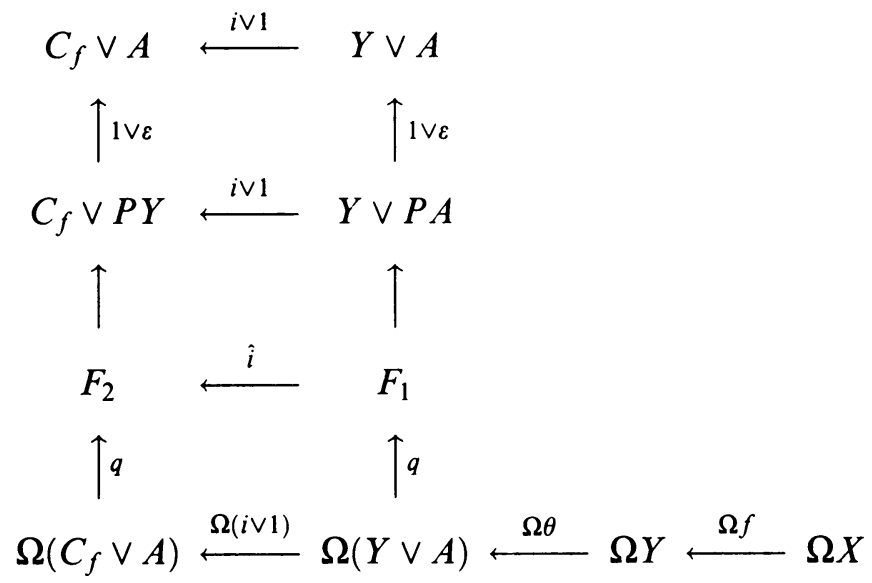

where $\hat{i}$ is induced by $i \vee 1, F_{1}, F_{2}$ are fibres of maps $1 \vee \varepsilon: Y \vee P A \rightarrow$ $Y \vee A, 1 \vee \varepsilon: C_{f} \vee P A \rightarrow C_{f} \vee A$ respectively.

Corollary $3.5[4$, Theorem 6]. Let $X$ be a co-H-space and $g: Y \rightarrow A$ cocyclic, that is, there is a map $\theta: Y \rightarrow Y \vee A$ such that $j \theta \sim(1 \times g) \Delta$, where $j:$ $Y \vee A \rightarrow Y \times A$ is the inclusion and $\Delta: Y \rightarrow Y \times Y$ is the diagonal map. Then there exists a map $\hat{\theta}: C_{f} \rightarrow C_{f} \vee A$ such that $p_{1} j^{\prime} \hat{\theta} \sim 1_{C_{f}}$ and $\hat{\theta} i=(i \vee 1) \theta$ if and only if $\hat{i} q \Omega(\theta f) \sim *$, where $j^{\prime}: C_{f} \vee A \rightarrow C_{f} \times A$ is the inclusion and $p_{1}: C_{f} \times A \rightarrow C_{f}$ is the projection.

Proof. From Theorem 3.4, it is sufficient to show that $\hat{i} q \Omega(\theta f) \sim *$ if and only if $(i \vee 1) \theta f \sim *$. If $(i \vee 1) \theta f \sim *$, then $\hat{i} q \Omega(\theta f) \sim q \Omega((i \vee 1) \theta f) \sim *$. Now suppose $\hat{i} q \Omega(\theta f) \sim *$. Since $F_{2} \rightarrow C_{f} \vee P A \stackrel{(1 \vee \varepsilon)}{\longrightarrow} C_{f} \vee A$ is a fibration, there is an exact sequence $\rightarrow\left[\Omega X, \Omega\left(C_{f} \vee P A\right)\right] \stackrel{\Omega(1 \vee \varepsilon)_{*}}{\longrightarrow}\left[\Omega X, \Omega\left(C_{f} \vee A\right)\right] \stackrel{q_{*}}{\rightarrow}\left[\Omega X, F_{2}\right] \rightarrow$ $\left[\Omega X, C_{f} \vee P A\right] \rightarrow$. Since $q_{*}(\Omega((i \vee 1) \theta f))=\hat{i} q \Omega(\theta f) \sim *$ and $\operatorname{Ker} q_{*}=$ $\operatorname{Im} \Omega(1 \vee \varepsilon)_{*}$, there is a map $\beta: \Omega X \rightarrow \Omega\left(C_{f} \vee P A\right)$ such that $\Omega(1 \vee \varepsilon) \beta \sim$ $\Omega((i \vee 1) \theta f)$. Since $X$ is a co- $H$-space, there is a map $s_{X}: X \rightarrow \Sigma \Omega X$ such that $e_{X} s_{X} \sim 1_{X}$, where $e_{X}=\tau^{-1}\left(1_{\Omega X}\right)$. Let $\nu=\tau^{-1}(\beta) s: X \rightarrow C_{f} \vee P A$. Since $\tau^{-1}(\beta)=e_{\left(C_{f} \vee P A\right)} \Sigma \beta$ and $\Omega e_{\left(C_{f} \vee P A\right)} \Omega \Sigma \beta=\beta \Omega e_{X}, \Omega \nu=\Omega\left(e_{\left(C_{f} \vee P A\right)} \Sigma \beta s_{X}\right)=$ $\beta \Omega\left(e_{X} s_{X}\right) \sim \beta$. Thus $\Omega((i \vee 1) \theta f) \sim \Omega(1 \vee \varepsilon) \beta \sim \Omega((1 \vee \varepsilon) \nu): \Omega X \rightarrow \Omega\left(C_{f} \vee A\right)$. Since $X$ is a co- $H$-space, the function $\Omega:\left[X, C_{f} \vee A\right] \rightarrow\left[\Omega X, \Omega\left(C_{f} \vee A\right)\right]$, given by $f \mapsto \Omega f$, is injective. Thus $(i \vee 1) \theta f \sim(1 \vee \varepsilon) \nu$. Let $l: C_{f} \vee P A \rightarrow$ $\left(C_{f} \vee A\right)^{I}$ be given by $l(z, *)=$ constant path at $(z, *)$ and by $l(*, \eta)=i_{2} \eta$, where $i_{2}: A \rightarrow C_{f} \vee A$ is the inclusion. Consider the map $h=l \nu: X \rightarrow$ $\left(C_{f} \vee A\right)^{I}$. Then $h: X \rightarrow\left(C_{f} \vee A\right)^{I}$ gives rise to a map $H: X \times I \rightarrow C_{f} \vee A$ with $H(x, t)=h(x)(t)$. Then $H(, 0)=(1 \vee *) \nu$ and $H(, 1)=(1 \vee \varepsilon) \nu$, where $*: P A \rightarrow A$ is the constant map. Thus $(i \vee 1) \theta f \sim(1 \vee \varepsilon) \nu \sim(1 \vee *) \nu=$ $i_{1} p_{1} j(1 \vee \varepsilon) \nu \sim i_{1} p_{1} j(i \vee 1) \theta f \sim i_{1} i f$, where $i_{1}: C_{f} \rightarrow C_{f} \vee A, j: C_{f} \vee A \rightarrow$ $C_{f} \times A$ are natural inclusions and $p_{1}: C_{f} \times A \rightarrow C_{f}$ is the projection. Thus we know, from Lemma 3.1, that $(i \vee 1) \theta f \sim *$.

We can also obtain the following proposition. The proof of Proposition 3.6 is similar to that of Theorem 3.4. So we will omit the proof. 
Proposition 3.6. Let $g: C_{f} \rightarrow A$ be a map such that $g i: Y \rightarrow A$ is cocyclic, that is, there is a map $\theta: Y \rightarrow Y \vee A$ such that $j \theta \sim(1 \times g i) \Delta$, where $j: Y \vee A \rightarrow$ $Y \times A$ is the inclusion. Then there exists a map $\hat{\theta}: C_{f} \rightarrow C_{f} \vee A$ such that $p_{1} j^{\prime} \hat{\theta} \sim 1_{C_{f}}, p_{2} j^{\prime} \hat{\theta} \sim g$, and $\hat{\theta} i=(i \vee 1) \theta$ if and only if $(i \vee 1) \theta f \sim *$, where $j^{\prime}: C_{f} \vee A \rightarrow C_{f} \times A$ is the inclusion and $p_{1}: C_{f} \times A \rightarrow C_{f}, p_{2}: C_{f} \times A \rightarrow A$ are projections.

Corollary 3.7. Let $[Y, A]=0$. Then, for any map $f: X \rightarrow Y,\left[C_{f}, A\right]=$ $D G\left(C_{f}, A\right)$.

Proof. Let $\theta: Y \rightarrow Y \vee A$ be given by $\theta(y)=(y, *)$. Then $(i \vee 1) \theta=i_{1} i$, where $i_{1}: C_{f} \rightarrow C_{f} \vee A$ is the inclusion. That is, $(i \vee 1) \theta: Y \rightarrow C_{f} \vee A$ can be extended to the map $i_{1}: C_{f} \rightarrow C_{f} \vee A$. Thus we know, from Lemma 3.1, that $(i \vee 1) \theta f \sim *$. Let $[g] \in\left[C_{f}, A\right]$. Since $i^{*}([g]) \in[Y, A]=0, g i \sim *$. Thus $j \theta \sim(1 \times *) \Delta \sim(1 \times g i) \Delta$ and $g i$ is cocyclic. From the fact $(i \vee 1) \theta f \sim *$ and Proposition 3.6, $[g] \in D G\left(C_{f}, A\right)$.

Remark 3.8. Consider the map $\phi: C_{f} \rightarrow \Sigma X \vee C_{f}$ given by $\phi(y)=(*, y)$, $\phi([x, t])=(\langle x, 2 t\rangle, *), 0 \leq t \leq 1 / 2, \phi([x, t])=(*,[x, 2 t-1]), 1 / 2 \leq t \leq 1$. Then the quotient map $q: C_{f} \rightarrow \Sigma X$ is cocyclic. Thus the induced map $q^{*}$ maps [ $\left.\Sigma X, A\right]$ into $D G\left(C_{f}, A\right)[8]$. Therefore, Corollary 3.7 can also be obtained from the Puppe sequence of the cofibration $Y \rightarrow C_{f} \rightarrow \Sigma X$.

\section{ACKNOWLEDGMENT}

I would like to thank Professor D. H. Gottlieb for helpful suggestions and the referee for his/her helpful suggestion.

\section{REFERENCES}

1. D. H. Gottlieb, Evaluation subgroups of homotopy groups, Amer. J. Math. 91 (1969), 729-756.

2. I. G. Halbhavi and K. Varadarajan, Gottlieb sets and duality in homotopy theory, Canad. J. Math. 27 (1975), 1042-1055.

3. H. B. Haslam, G-spaces and H-spaces, Ph.D. dissertation, Univ. of California, Irvine, 1969.

4. C. S. Hoo, Lifting Gottlieb sets, J. London Math. Soc. (2) 14 (1976), 535-544.

5. K. L. Lim, On cyclic maps, J. Austral. Math. Soc. Ser. A 32 (1982), 349-357.

6. R. M. Switzer, Algebraic topology-homotopy and homology, Springer-Verlag, New York, 1975.

7. K. Tsuchida, Principal cofibrations, Tohuku Math. J. 16 (1964), 321-333.

8. K. Varadarajan, Generalized Gottlieb groups, J. Indian Math. Soc. 33 (1969), 141-164.

Department of Mathematics, Hannam University, Taejon, 300-791, Korea 